\title{
ANTICOMMUTANT LIFTING AND ANTICOMMUTING DILATION
}

\author{
ZOLTÁN SEBESTYÉN
}

(Communicated by Palle E. T. Jorgensen)

\begin{abstract}
In this paper we prove the anticommutant counterpart of the Sz.Nagy-Foias commutant lifting theorem and Ando's theorem on unitary dilation of a pair of commutative contractions. A dual extension theorem applies in the one-step procedure of our approach.
\end{abstract}

\section{INTRODUCTION}

One of the best known results in dilation, extension, and interpolation theory is the Sz.-Nagy-Foias commutant lifting theorem [8]. Ando's theorem [1] on commuting unitary dilation of a pair of commutative contractions is also a consequence of the theorem mentioned above [3, p. 321].

In Theorems 2 and 3 we prove the anticommutant counterparts of the Sz.Nagy-Foias commutant lifting theorem. Our proof includes both variants.

Our approach applies each step of the dual extension version of Parrot's theorem [3, Theorem 1], one of the neatest results in extension, dilation, and interpolation theory (see, e.g., $[2,4,5])$.

\section{Dual extension}

We formulate here a dual extension version of Parrott's theorem on quotient norms [3, Theorem 1] in a different way than Foiaş and Tannenbaum did [2, Theorem 1] (see [4, Theorem] or [5, Theorem 1]).

Theorem 1. Let $K$ and $K^{\prime}$ be Hilbert spaces, $H \subset K$ and $H^{\prime} \subset K^{\prime}$ be subspaces, and $X: H \rightarrow K^{\prime}$ and $X^{\prime}: H^{\prime} \rightarrow K$ be given bounded linear transformations. Then there exists operator $Y: K \rightarrow K^{\prime}$ extending $X$ so that $Y^{*}$ extends $X^{\prime}$ if and only if the following identity holds true:

$$
\left(X h, h^{\prime}\right)=\left(h, X^{\prime} h^{\prime}\right) \quad\left(h \in H, h^{\prime} \in H^{\prime}\right) .
$$

Moreover $Y$ can be of norm $\max \left\{\|X\|,\left\|X^{\prime}\right\|\right\}$ possible at most.

Proof. Reduction to a selfadjoint norm-preserving extension theorem of Krein will be as follows. Given the symmetric operator $S: H \oplus H^{\prime} \rightarrow K \oplus K^{\prime}$ by

$$
S\left(h ; h^{\prime}\right):=\left(X^{\prime} h^{\prime} ; X h\right) \quad\left(h \in H, h^{\prime} \in H^{\prime}\right)
$$

Received by the editors October 28, 1991 and, in revised form, August 10, 1992.

1991 Mathematics Subject Classification. Primary 47A20; Secondary 93C05.

Key words and phrases. Sz.-Nagy-Foias commutant lifting, Parrott's theorem, Ando's theorem, dilation theory, one-step extension, anticommuting contractions and unitaries. 
we see that $\|S\|=\max \left\{\|X\|,\left\|X^{\prime}\right\|\right\}$. So $S$ has selfadjoint extension $\tilde{S}$ to $K \oplus K^{\prime}$ such that $\|\widetilde{S}\|=\|S\|$. Then $Y=P^{\prime} \widetilde{S} P^{*}$ is a solution of this dual extension problem with extremal (least) norm where $P$ and $P^{\prime}$ are the natural projections of $K \oplus K^{\prime}$ onto $K$ and $K^{\prime}$, respectively. The extension properties of $Y$ are plain and the norm of $Y$ not greater than $\|\widetilde{S}\|=\max \left\{\|X\|,\left\|X^{\prime}\right\|\right\}$ on one side but at least such otherwise.

\section{3. (ANTI-)COMMUTANT LIFTING}

Given a Hilbert space $H$, an operator $S$ on $H$ will be called a contraction if its norm $\leq 1$. And the operator $X_{0}$ on $H$ belongs to the commutant or anticommutant of $S$ if $X_{0}$ satisfies for $\mathscr{E}=1$ or -1 , respectively,

$$
S X_{0}=\mathscr{E} X_{0} S .
$$

Now let $U$ be the minimal isometric dilation of $S$ acting on a Hilbert space $K$, containing $H$ as a subspace. We know $[3,8]$ that $U^{*}$ extends $S^{*}$ and $K=\bigcup_{n=0}^{\infty} K_{n}$ with $K_{n}=\bigvee_{k=0}^{n} U^{k}(H)$ (thus $K_{0}=H$ ), moreover the orthogonal projections $P_{n}: K \rightarrow K_{n}$ satisfy $P_{n+1} U=U P_{n}$ for any natural $n$.

Let $X_{0}$ be an anticommutant or commutant operator of $S$. We lift $X_{0}$ to $K$ in the minimal unitary dilation space.

Theorem 2. Let $S$ be a contraction and $X_{0}$ be an operator on the Hilbert space $H$. Assume identity (1). If $U$ is the minimal isometric dilation of $S$ acting on the Hilbert space $K$ then there exists an operator $X_{\mathscr{E}}$ on $K$ such that $X_{\mathscr{E}}^{*}$ extends $X_{0}^{*},\left\|X_{\mathscr{E}}\right\|=\left\|X_{0}\right\|$ and

$$
U X_{\mathscr{E}}=\mathscr{E} X_{\mathscr{E}} U
$$

Proof. At the first step we find $X_{1}: K_{1} \rightarrow K_{1}$ with $\left.X_{1}^{*}\right|_{K_{0}}=X_{0}^{*},\left.X_{1}\right|_{H_{0}}=$ $\left.\mathscr{E} U X_{0} U^{*}\right|_{H_{0}}$, where $H_{0}=U(H)$, and such that $\left\|X_{1}\right\|=\left\|X_{0}\right\|$ and $\mathscr{E} U X_{0} P_{0}=$ $X_{1} P_{1} U$. To do this we should check the adjoining identity of Theorem 1: for $h, k \in H_{0}$

$$
\begin{aligned}
\left(\mathscr{E} U X_{0} U^{*}(U h), k\right) & =\mathscr{E}\left(X_{0} h, U^{*} k\right)=\mathscr{E}\left(X_{0} h, S^{*} k\right)=\mathscr{E}\left(S X_{0} h, k\right) \\
& \stackrel{(1)}{=} \mathscr{E}^{2}\left(X_{0} S h, k\right)=\left(P_{0} S h, X_{0}^{*} k\right)=\left(U h, X_{0}^{*} k\right) .
\end{aligned}
$$

This solution then satisfies

$$
\mathscr{E} U X_{0} P_{0}=\mathscr{E} U X_{0} U^{*}\left(U P_{0}\right)=X_{1}\left(U P_{0}\right)=X_{1} P_{1} U
$$

The second step yields $X_{2}: K_{2} \rightarrow K_{2}$ such that $\left.X_{2}^{*}\right|_{K_{1}}=X_{1}^{*},\left.X_{2}\right|_{H_{1}}=$ $\left.\mathscr{E} U X_{1} U^{*}\right|_{H_{1}}$, where $H_{1}=U\left(K_{1}\right)$, and moreover $\left\|X_{2}\right\|=\left\|X_{1}\right\|$ and $\mathscr{E} U X_{1} P_{1}=$ $X_{2} P_{2} U$. That the adjoining identity satisfies in this setting is less obvious:

$$
\begin{aligned}
\left(\mathscr{E} U X_{1} U^{*}(U h), k\right) & =\mathscr{E}\left(P_{1} U X_{1} h, k\right)=\mathscr{E}\left(U P_{0} X_{1} h, k\right) \\
& =\mathscr{E}\left(h, X_{1}^{*} P_{0} U^{*} k\right)=\mathscr{E}\left(h, X_{0}^{*} P_{0} U^{*} k\right) \\
& =\left(\mathscr{E} U X_{0} P_{0} h, k\right)=\left(X_{1} P_{1} U h, k\right) \\
& =\left(U h, P_{1} X_{1}^{*} k\right)=\left(U h, X_{1}^{*} k\right)
\end{aligned}
$$

holds indeed for any $h, k \in K_{1}$.

In the $n$th step we argue similarly to have $X_{n}: K_{n} \rightarrow K_{n}$ with $\left.X_{n}^{*}\right|_{K_{n-1}}=$ $X_{n-1}^{*},\left.\quad X_{n}\right|_{H_{n-1}}=\left.\mathscr{E} U X_{n-1} U^{*}\right|_{H_{n-1}}$, where $H_{n-1}=U\left(K_{n-1}\right)$, and $\left\|X_{n}\right\|=$ $\left\|X_{n-1}\right\|, \mathscr{E} U X_{n-1} P_{n-1}=X_{n} P_{n} U$. 
The necessary change in checking is that one writes $n-1$ and $n-2$ instead of 1 and 0 , respectively, used at the 2 nd step. As a result of this process, thanks to the minimality of the dilation, we arrive at an operator $X_{\mathscr{E}}^{*}: K \rightarrow K$ as the extension of each of $\left\{X_{n}^{*}\right\}_{n=0}^{\infty}$, with norm $\left\|X_{0}\right\|$. Since then $X_{n} P_{n}$ converges to $X_{\mathscr{E}}$ in the strong operator topology, the identity $\mathscr{E} U X_{n-1} P_{n-1}=X_{n} P_{n} U$ gives the desired identity (2). Thus $X_{0}$ is lifted to a desired dilation $X_{\mathscr{E}}$ on $K$.

Theorem 3. Let $S$ be a contraction and $X_{0}$ be an operator on the Hilbert space $H$ with identity (1). If $U$ is the minimal unitary dilation of $S$ acting on a Hilbert space $K$ then there exists an operator $X_{\mathscr{E}}$ on $K$ which is the dilation of $X_{0},\left\|X_{\mathscr{E}}\right\|=\left\|X_{0}\right\|$, and identity (2) also holds true.

Proof. First let $U_{+}$be the minimal isometric dilation of $S$ acting on a Hilbert space $K_{+}$that lies between $H$ and $K$. An application of Theorem 2 gives a dilation $X_{\mathscr{E}+}$ of $X$ to $K_{+}$with $\left\|X_{\mathscr{E}+}\right\|=\|X\|$ and the corresponding identity holds:

$$
U_{+} X_{\mathscr{E}+}=\mathscr{E} X_{\mathscr{E}+} U_{+}
$$

Now, as $U^{*}$ is the minimal isometric ailation of $U_{+}^{*}$, identity $\left(2_{+}\right)$reads as follows:

$$
U_{+}^{*} X_{\mathscr{E}+}^{*}=\mathscr{E} X_{\mathscr{E}+}^{*} U_{+}^{*} .
$$

Another application of Theorem 2 yields an operator $X_{\mathscr{E}}^{*}$ on $K$ that extends $X_{\mathscr{E}+},\left\|X_{\mathscr{E}}\right\|=\left\|X_{\mathscr{E}+}\right\|=\left\|X_{0}\right\|$, and identity (2) satisfies. The proof is complete.

\section{4. (ANTI-)COMMUTING DILATION}

The situation changes to a pair of contractions $S$ and $T$ on a Hilbert space $H$, which satisfies

$$
S T=\mathscr{E} T S, \quad \text { where } \mathscr{E}=-1 \text { or } 1 .
$$

In other words $S, T$ are (anti-)commuting operators if $\mathscr{E}$ equals $(-1)$ or 1. Ando's theorem [1] ensures for the $\mathscr{E}=1$ case a commuting pair of unitaries $U, V$ on a larger Hilbert space so that $U$ and $V$ are dilations of $S$ and $T$, respectively. Here we prove the similar statement for anticommuting contractions and unitaries as well.

Theorem 4. Let $S$ and $T$ be (anti-)commuting contractions on a Hilbert space $H$. Then there exists an (anti-)commuting pair of unitaries $U$ and $V$ that are common unitary dilations of $S$ and $T$, respectively.

Proof. An application of Theorem 3 yields a dilation of the pair $S, T$, say, $U_{0}, T_{0}$, respectively, acting on a Hilbert space $K_{0}$ so that $U_{0}$ is the minimal unitary dilation of $S$, and $T_{0}$ is a contractive dilation of $T$ to $K_{0}$. with

$$
U_{0} T_{0}=\mathscr{E} T_{0} U
$$

Then let $V$ be the minimal unitary dilation of $T_{0}$ that acts on a Hilbert space $K$. Our goal is to extend $U_{0}$ to a unitary operator $U$ on $K$ so that

$$
U V=\mathscr{E} V U \text {. }
$$

Due to the minimality assumption $K=\bigvee_{n=-\infty}^{\infty} V^{n}\left(K_{0}\right)$ the operator $U$ has to be defined on $V^{n} k_{0}$ for $n \in \mathbb{Z}$ and $k_{0} \in K_{0}$ as $\mathscr{E}^{n} V^{n} U_{0} k_{0}$. Doing so, $U$ is 
seen to be an onto map. That $U$ is isometric, hence unitary, follows by the identity (if $m \geq n$ )

$$
\begin{aligned}
\left(\mathscr{E}^{m} V^{m} U_{0} h_{0}, \mathscr{E}^{n} V^{n} U_{0} k_{0}\right) & =\mathscr{E}^{m+n}\left(V^{m-n} U_{0} h_{0}, U_{0} k_{0}\right) \\
& =\mathscr{E}^{m+n}\left(T_{0}^{m-n} U_{0} h_{0}, U_{0} k_{0}\right) \\
& =\mathscr{E}^{m+n} \mathscr{E}^{m-n}\left(U_{0} T_{0}^{m-n} h_{0}, U_{0} k_{0}\right) \\
& =\left(T_{0}^{m-n} h_{0}, k_{0}\right)=\left(V^{m} h_{0}, V^{n} k_{0}\right),
\end{aligned}
$$

where $m, n \in \mathbb{Z}, h_{0}, k_{0} \in K_{0}$, are arbitrary. Remark that $K_{0}$ reduces $U$ as well. Our final goal is to prove

$$
S^{m} T^{n}=\left.P_{H} U^{m} V^{n}\right|_{H} \quad(m, n=0,1,2, \ldots),
$$

where $P_{H}$ stands for the orthogonal projection of $K$ to $H$. First we know that

$$
S^{m} T^{n}=\left(\left.P_{H} U_{0}^{m}\right|_{H}\right)\left(\left.P_{H} T_{0}^{n}\right|_{H}\right)=\left.P_{H} U_{0}^{m} T_{0}^{n}\right|_{H},
$$

as in the proof of [9, Theorem 6]. Hence we have that for $h_{0}, k_{0} \in K_{0}$ and $m, n \in N$

$$
\begin{aligned}
\left(U_{0}^{m} T_{0}^{n} h_{0}, k_{0}\right) & =\left(T_{0}^{n} h_{0}, U_{0}^{* m} k_{0}\right)=\left(V^{n} h_{0}, U_{0}^{* m} k_{0}\right) \\
& =\left(V^{n} h_{0}, U^{* m} k_{0}\right)=\left(U^{m} V^{n} h_{0}, k_{0}\right) .
\end{aligned}
$$

This implies that the (anti-)commuting pair of unitaries $U, V$ are common dilations of the pair of contractions $S, T$ indeed.

\section{REFERENCES}

1. T. Ando, On a pair of commutative contractions, Acta Sci. Math. (Szeged) 24 (1963), 88-90.

2. C. Foiaş and A. Tannenbaum, A strong Parrot theorem, Proc. Amer. Math. Soc. 106 (1989), 777-784.

3. S. Parrott, On the quotient norm and the Sz.-Nagy-Foias lifting theorem, J. Funct. Anal. 30 (1978), 3i1-328.

4. Z. Sebestyén, A proof of Parrott's theorem on quotient norms, Period Math. Hungar. 20 (1989), 85-87.

5. __ Parrott's theorem versus strong Parrott theorem, Semesterberichte Funktionalanalysis Tübingen, Wintersemester 89/90, pp. 227-229.

6. Z. Sebestyén and Á. Magyar, Compact self-adjoint extensions of suboperators, Linear Algebra Appl. (submitted).

7. B. Sz.-Nagy and C. Foiaş, Dilation des commutants d'operateurs, C. R. Acad. Sci. Paris Sér. I Math. 266 (1968), 493-495.

8. _ Harmonic analysis of operators on Hilbert space, North-Holland, Amsterdam, 1970.

9. R. G. Douglas, P. S. Muhly, and C. Pearcy, Lifting commuting operators, Michigan Math. J. 15 (1968), 385-395.

Department of APPlied analysis, Eötvös University, 1088 Budapest, MúzeUm KRT. 6-8, HUNGARY

E-mail address: sebestyenzol@ludens.elte.hu 